\title{
GENETIC ANALYSIS OF THE GENUS DIOSPYROS SSP. USING RAPD AND I-PBS METHODS
}

\author{
J. Raddová, H. Ptáčková, J. Čechová, I. Ondrášek \\ Received: September 13, 2012
}

\begin{abstract}
RADDOVÁ, J., PTÁČKOVÁ, H., ČECHOVÁ, J., ONDRÁŠEK, I.: Genetic analysis of the genus Diospyros ssp. using RAPD and i-PBS methods. Acta univ. agric. et silvic. Mendel. Brun., 2012, LX, No. 8, pp. 205-216

Molecular techniques (RAPD and i-PBS) were used to study genetic diversity within persimmon collection at Horticulture Faculty of Mendel University in Lednice. The aim of the work was to distinguish 14 known and 6 of unknown origin persimmon cultivars. The basic screening of 20 OPT primers was applied to 4 cultivars differring in the place of origin. Within the group of screened primers there were chosen those, which gave polymorphic repeatable strong and middle strong bands. Selected primers were used for the RAPD reactions within the whole persimmon collection. Three OPA primers previously described in the literature were also used for the RAPD reactions within the whole persimmon collection. Additional 16 i-PBS primers previously described in the literature were also used for i-PBS analysis of the whole group of cultivars. Amplification was successful with 12 i-PBS primers. The FreeTree software package was used to generate a similarity matrix and then to produce a dendrogram using UPGMA analyses. The similarity dendrograms of all persimmon cultivars were created based on both approaches and also on combination of both analyses by program Tree View. All the dendrograms clearly separated the assessed cultivars into 4 clusters. There are cluster of American persimmons - Meader' (1), 'Garretson' (2) and 'Early Golden' (3). They are representatives of D. virginiana. Further part of dendrogram includes single D. lotus (5), which is also clearly separated from other cultivars of the genus Diospyros. The third cluster includes interspecific hybrids 'Rossiyanka' (10) and 'Nikitskaiya Bordovaiya' (13), which arised from crosses of D. virginiana and D. kaki. The last cluster is formed by cultivars of Japanese persimmon - 'Mikatani Gosho', 'Zenjimaru', 'Tone Wase', 'Hiratanenashi', 'Fuyu', Chinese cultivar - 'Sansi' and two Italian cultivars 'Vaniglia' and 'Tipo'. They are clustered without significant distinction. The similarities and the differences revealed among incorporation of cultivars into groups were compared with the literature findings.
\end{abstract}

persimmon, RAPD, i-PBS, primer, dendrogram

Persimmon belongs to important fruit cultures. Persimmon is mainly cultivated in the subtropical areas nevertheless some very early ripenig cultivars and interspecific cultivars can be grown under climate conditions in the Czech Republic (Ondrášek and Krška, 2009). The largest distributions are in the countries of its origin such as China, Japan and Korea. China is the biggest world producer of persimmon and Italy is major European producer of persimmon (Faostat, 2009).

There are three the most important species of Diospyros, namely, Diospyros kaki Thunb. (Japanese persimmon), Diospyros virginiana L. (American persimmon) and botanical species Diospyros lotus L. Diospyros kaki is the most important species from commercial point of view (Yonemori et al., 2000; Giordani, 2002). Japanese persimmon can be classified into four types based on the relationship among astringency in the fruit at harvest, presence of seed, and flesh colour, i.e., pollination constant and non-astringent (PCNA), pollination variant and non-astringent (PVNA), pollination variant and astringent (PVA), pollination constant and astringent (PCA) (Sugiura, 2005). 
More than 900 cultivars are known nowadays. Identification of persimmon cultivars is one of the main problems in the present due to misleading transliterations, local names, synonyms and homonyms. Cases of synonymies and homonyms do exist accessions collected in different sites, and possibly the names of a given genotype do not correspond to the denomination of the true to type accession held by reference germplasm collections of the countries of origin (namely Japan). At the European level 37\% of accessions are positively identified, while the status of identification of the remaining $63 \%$ is probable $(55 \%)$ and totally uncertain (8\%) (Bellini and Giordani, 2003).

For characterization of germplasm it is essential to identify individual genotypes. Therefore one of the important aspects of fruit growing is the reliable determination of grown cultivars (Giordani, 2002; Sugiura, 2005; Yakushiji and Nakatsuka, 2007). Authenticating the identity of germplasm resources of persimmon would be a great value for breeding (Badenes et al., 2003).

There are various methods for cultivar identification and distinctness. First, there are methods based on morphological traits, and biochemical markers as analysis of isozymes (Mondini et al., 2009). Limiting factors of these techniques for cultivar identification led to the development of methods based on polymerase chain reaction (PCR) (Akbulut et al., 2008).

Therefore to identify different varieties of persimmon there are nowadays applied techniques based on restriction fragment length polymorphisms (RFLP) (Nakamura and Kobayashi, 1994), random amplification of polymorphic DNA (RAPD) (Thaipong et al., 2003; Yamagishi et al., 2005; Yýldýz, et al., 2007; Akbulut et al., 2008; Zahng et al., 2009), amplified fragment length polymorphism (AFLP) (Yonemori et al., 2008) or single sequence repeat (SSR) (Guo and Luo, 2011).

RAPD markers have been used successfully for estimating genetic relationships in several fruit crops such as apple (Koller et al., 1993), peach (Badenes et al., 1998), almond (Bartolozzi et al., 1998), plum (Boonprakob et al., 2001), guava (Prakash et al., 2002), grapevine (Ulanovsky et al., 2002), apricot (Ercisli et al., 2009), citrus fruit (Ji et al., 2011) and also persimmon (Thaipong et al., 2003; Bellini et al., 2003). The RAPD method seems to be a very effective indicator of different persimmon cultivars (Nakamura and Kobayashi, 1994).

One recently described retrotransposon-based molecular marker technique was used to identify the varieties (Mondini et al., 2009). There are several retrotransposon-based molecular methods which require sequence information to design elementspecific primers (Kalendar, 2011; Kalendar et al., 2011). Retrotransposons are kind of elements which can be found in the genome of many eukaryotic organisms (Kumar and Bennetzen, 1999). One retrotransposon-based molecular method does not require previous sequence knowledge, it's i-PBS (inter-primer binding site). I-PBS primers were designed according the highly conservative sequences called Primer Binding Site (PBS) to which tRNA binds, acting as a primer for reverse transcriptase during the replication cycle of retroviruses and LTR retrotransposons. I-PBS is a universal and efficient method from identification of polymorphism (Kalendar et al, 2010; Kalendar, 2011; Kalendar et al., 2011).

The aim of this paper was to differentiate persimmon collection by means of RAPD and i-PBS methods, study genetic diversity within persimmon collection and to incorporate a unknown persimmon cultivars in the dendrogram.

\section{MATERIAL AND METHODS}

\section{Plant materials}

Twenty cultivars of persimmon (14 known cultivars and 6 unknown cultivars, see Tab. I) was included in this study. All plant material was obtained from a local persimmon collection situated in Lednice in the premises of Horticulture Faculty, Mendel University, Czech Republic.

\section{DNA Extraction}

Total genomic DNA was extracted from young frozen leaves $(0.1 \mathrm{~g})$ using DNeasy Plant Mini Kit (Qiagen). The DNA quality and concentration was determined by means of electrophoresis on a $0.8 \%$ agarose gel compared with lambda DNA standards containing 200 and $400 \mathrm{ng} / \mu \mathrm{l}$ DNA. The DNA concentration was also determined by fluorometr Modulus (Turner BioSystems).

\section{RAPD Analysis}

The RAPD amplification was performed in volume of $25 \mu \mathrm{l}$ containing $\mathrm{H}_{2} \mathrm{O}$, $1 \times$ PCR buffer (Finnzymes), $100 \mu \mathrm{M}$ each of dNTPs (Promega), $0.4 \mu \mathrm{M}$ 10-mer primer (Operon), 1 unit of Taq polymerase (Finnzymes) and $20 \mathrm{ng}$ of template DNA. DNA amplifications were performed in a thermal cycler UNO II (Biometra), according to the program of Williams et al. (1990) and the program used in the Mendeleum Research Station (Raddová, 2005). The program consisted of: denaturation at 94 ${ }^{\circ} \mathrm{C}$ for $3 \mathrm{~min}$, followed by 40 cycles (denaturation at $94^{\circ} \mathrm{C}$ for $20 \mathrm{~s}$, annealing at $36^{\circ} \mathrm{C}$ for $1 \mathrm{~min}$, extension at $72{ }^{\circ} \mathrm{C}$ for $1 \mathrm{~min}$ ), finishing at $72{ }^{\circ} \mathrm{C}$ for $9 \mathrm{~min}$. The amplified products were separated by horizontal electrophoresis Agagel Maxi (Biometra@) in 1.5\% agarose gel and visualised by GelRed.

At first, screening of 4 cultivars different in the place of origin ('Early Golden', 'Mikatani Gosho', 'Sansi', 1115 KOK) by using 20 OPT primers (OPT-1 to OPT-20) was performed. RAPD polymorphic bands were scored as 1 for presence of a band and 0 for its absence and were transformed into a binary matrix. Data from screening were transferred to software Popgen (Yeh and Boyle, 1997) and then the primers were evaluated with the highest genetic 
I: Persimmon cultivars used in this study

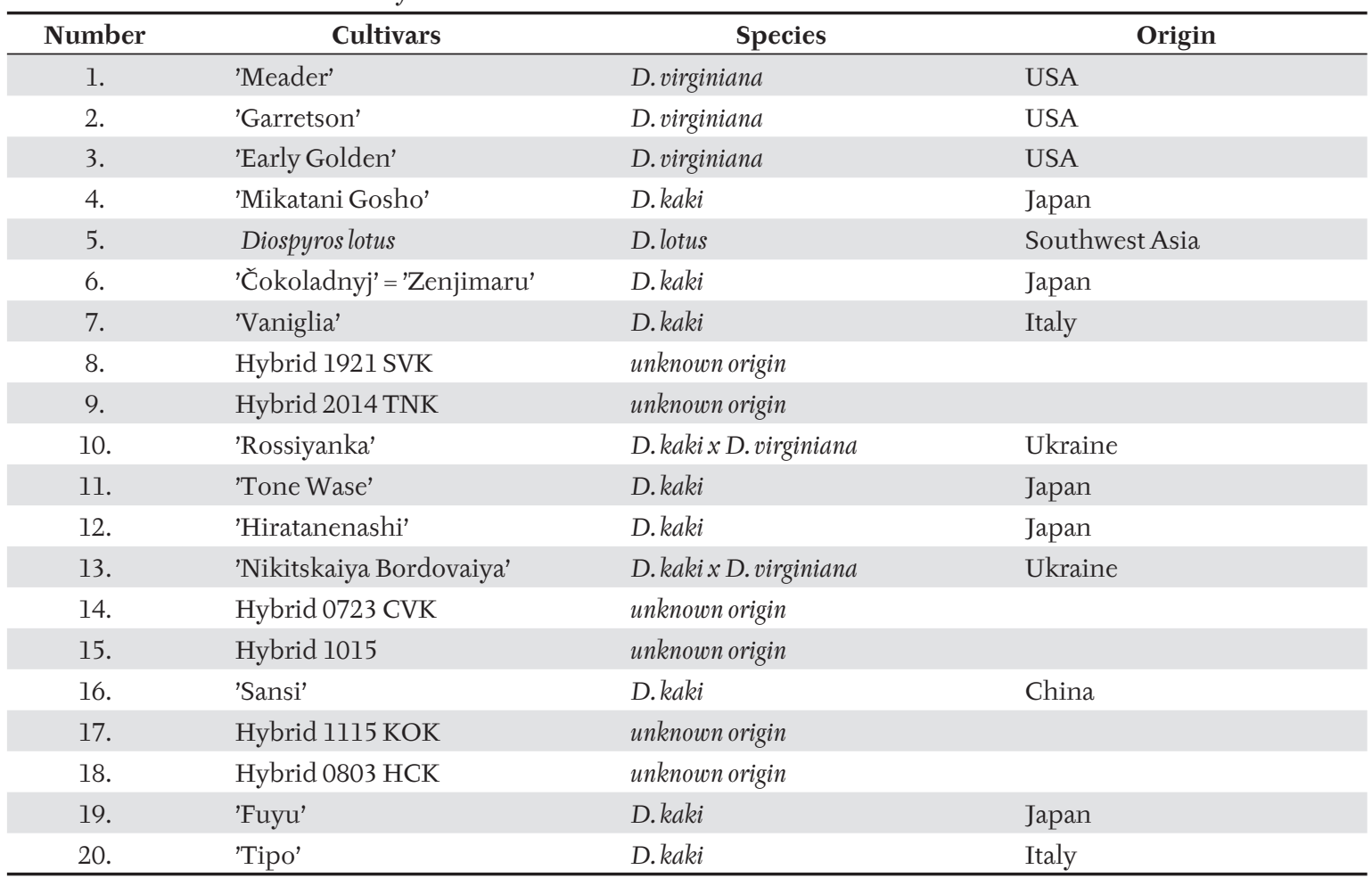

diversity (Nei, 1972). The OPT primers which produced polymorphic repetable strong bands were afterwards used in RAPD analysis of whole group of cultivars. At the same time analysis of whole group of cultivars was performed by using 3 primers from kit OPA (OPA-8, OPA-18, OPA-19) which successfully used Badenes et al. (2003).

Similarity among all cultivars was estimated according to unweighted pair group method average (UPGMA). The FreeTree software package (Hampl et al., 2001) was used to generate a mean character difference matrix/similarity matrix and then to produce a dendrogram using UPGMA analyses. Final dendrogram was constructed by program Tree View (Page, 1996).

\section{I-PBS technique}

The i-PBS amplification according to work of Kalendar et al. (2010) was performed in volume of $25 \mu$ l containing $\mathrm{H}_{2} \mathrm{O}, 1 \times$ PCR buffer, $1 \mu \mathrm{M}$ 12-mer primer (i-PBS primers), $0.2 \mathrm{mM}$ dNTPs (Promega), 1.25 unit of Taq polymerase (Finnzymes) and $20 \mathrm{ng}$ of template DNA. After denaturation at $95^{\circ} \mathrm{C}$ for 3 min, DNA amplifications were performed for 29 cycles in a UNO II thermal cycler (Biometra), according to the program of Kalendar et al. (2010). The PCR program consisted of: 1 cycle at $95^{\circ} \mathrm{C}$ for $3 \mathrm{~min}$; 28-30 cycles of $95^{\circ} \mathrm{C}$ for $15 \mathrm{~s}, 50-60{ }^{\circ} \mathrm{C}$ (Kalendar et al., 2010) for $60 \mathrm{~s}$, and $68{ }^{\circ} \mathrm{C}$ for $60 \mathrm{~s}$; a final extension step of $72{ }^{\circ} \mathrm{C}$ for $5 \mathrm{~min}$. Also 16 i-PBS primers (2074, 2076, 2077, 2078, 2270, 2271, 2272, $2273,2374,2376,2377,2378,2385,2386,2389,2394)$ were used for analysis of whole group of cultivars.
The amplified products were separated by horizontal electrophoresis Agagel Maxi (Biometra@) in $1.5 \%$ agarose gel and visualised by GelRed. I-PBS polymorphic bands were scored as 1 for presence of a band and 0 for its absence and were transformed into a binary matrix. The FreeTree software package (Hampl et al., 2001) was used to generate a mean character difference matrix/similarity matrix and then to produce a dendrogram using UPGMA analyses. Final dendrogram was constructed by program Tree View (Page, 1996).

\section{RESULTS}

\section{OPT primer screening}

A set of 20 OPT primers was used for screening of 4 cultivars. Primers generating a higher genetic polymorphism were chosen. Nei's (1972) gene diversity between cultivars achieved an average of 0.325. Out of 20 primers tested, 5 of them were not possible to evaluable, 1 of them amplified nonpolymorphic product and 14 primers amplified from 2 to 12 polymorphic bands ranged from 200 to 2200 bp. The number of polymorphic markers was 83 (82.93\%) out of the total number of 328 bands.

Consequently, 10 OPT primers (OPT-01, OPT-03, OPT-09, OPT-11, OPT-12, ОРТ-16, ОРТ-17, ОРТ-18, OPT-19, OPT-20) were selected for further work after screening, visual and statistical evaluation. These 10 OPT primers were used for analysis of the whole collection of 20 persimmon cultivars. 


\section{RAPD analysis used for all cultivars}

The group of RAPD primers selected during the basic screening distinguished all the analysed cultivars. The OPT primers created a total of 1140 polymorphic bands scored in 57 markers. Selected primers generated from 3 to 11 polymorphic products in the size range of $220 \mathrm{bp}$ to $2100 \mathrm{bp}$. The OPA primers made a total of 620 polymorphic bands scored in 31 markers. Each primer generated from 7 to 15 polymorphic bands. The size of the amplified fragments ranged from $150 \mathrm{bp}$ to $1400 \mathrm{bp}$.

In total, 1760 polymorphic bands in 88 markers were generated by RAPD primers (OPT and OPA) and the size of the amplified fragments ranged from $150 \mathrm{bp}$ to $2100 \mathrm{bp}$. The OPA primers reached an average yield of 10.3 markers/primer and the OPT primers had an average yield of 5.7 markers/primer.

The example of image - Fig. 1 shows electrophoretic spectrum of analysed cultivars. Numbers 1-20 mean numbers of cultivars. The letter " $\mathrm{M}$ " indicates the 100 bp DNA ladder (Life Technologies). Cultivars like: 'Meader' (1), 'Garretson' (2), 'Early Golden '(3), botanical species Diospyros lotus (5), 'Rossiyanka' (10) and 'Nikitskaiya Bordovaiya' (13) have similar spectrum of products and were significantly distinguished from others, in the Fig. 1. Further cultivars 'Tone Wase' (11) and 'Hiratanenashi' (12) have similar spectrum of products and were significantly distinguished from other cultivars too.

The dendrogram of genetic relationships among persimmon cultivars based on RAPD primers is presented in Fig. 2. The RAPD dendrogram classified all the 20 cultivars into four groups. Cluster analysis grouped the cultivars as follows.

First is a cluster of American persimmons, where 3 cultivars of American persimmon are included: 'Meader' (1), 'Garretson' (2) and 'Early Golden' (3). They are representatives of D. virginiana. This cluster was clearly separated from the rest of cultivars, while cultivars 'Meader' (1) and 'Early Golden' (3) were not distinguished using the RAPD markers.

The second part of dendrogram includes single botanical species Diospyros lotus (5), which is also clearly separated from other cultivars of the genus Diospyros.

The third cluster includes interspecific hybrids 'Rossiyanka' (10) and 'Nikitskaiya Bordovaiya' (13), which arised from crosses of D. virginiana and D. kaki. The remaining and largest part creates a cluster of cultivars without significant distinction. This cluster includes fourteen cultivars belonging to the species D. kaki. Cultivars of unknown origin 1115 KOK (17), 1015 (15), 1921 SVK (8), 2014, TNK (9), 0723 CVK (14) and 0803 HCK (18) are in the same cluster like known cultivars 'Zenjimaru' (6), 'Vaniglia' (7), 'Sansi' (16), 'Tipo' (20), 'Hiratanenashi' (12),'Tone Wase' (11), 'Mikatani Gosho' (4) and 'Fuyu' (19) and therefore these cultivars share similar genetic background.

\section{I-PBS analysis}

Sixteen i-PBS primers were used for analysis of the whole group of cultivars. The amplification was successful with twelve i-PBS primers (primers: 2076 , 2077, 2078, 2272, 2273, 2374, 2376, 2377, 2378, 2386, 2389, 2394). Four i-PBS primers (primers: 2074, $2270,2271,2385)$ it was not possible to evaluate, even after repeating.

Twelve i-PBS primers created a total of 1760 polymorphic bands scored in 88 markers and generated distinctive 4 to 12 polymorphic bands products in the size range of $100 \mathrm{bp}$ to $2000 \mathrm{bp}$. Primers i-PBS reached an average yield of 7.3 markers/primer.

The example of image - Fig. 3 shows electrophoretic spectrum of analysed cultivars. Numbers 1-20 mean numbers of cultivars. The letter " $\mathrm{M}$ " indicates the 100 bp DNA ladder (Life Technologies). There is the name of the primer and annealing temperature in the right corner of

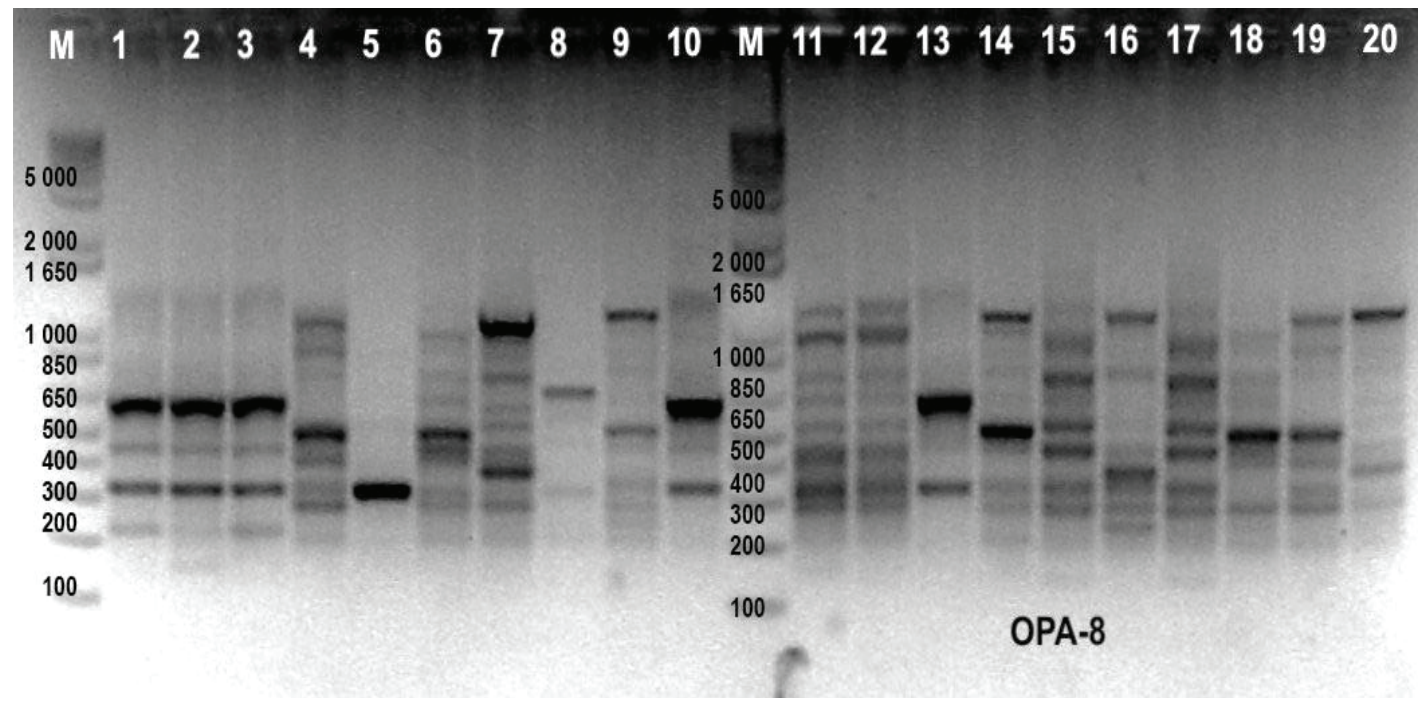

1: RAPD analysis with OPA-8 primer, 1-20 - numbers of cultivars, "M" - 100 bp DNA ladder (Life Technologies) 


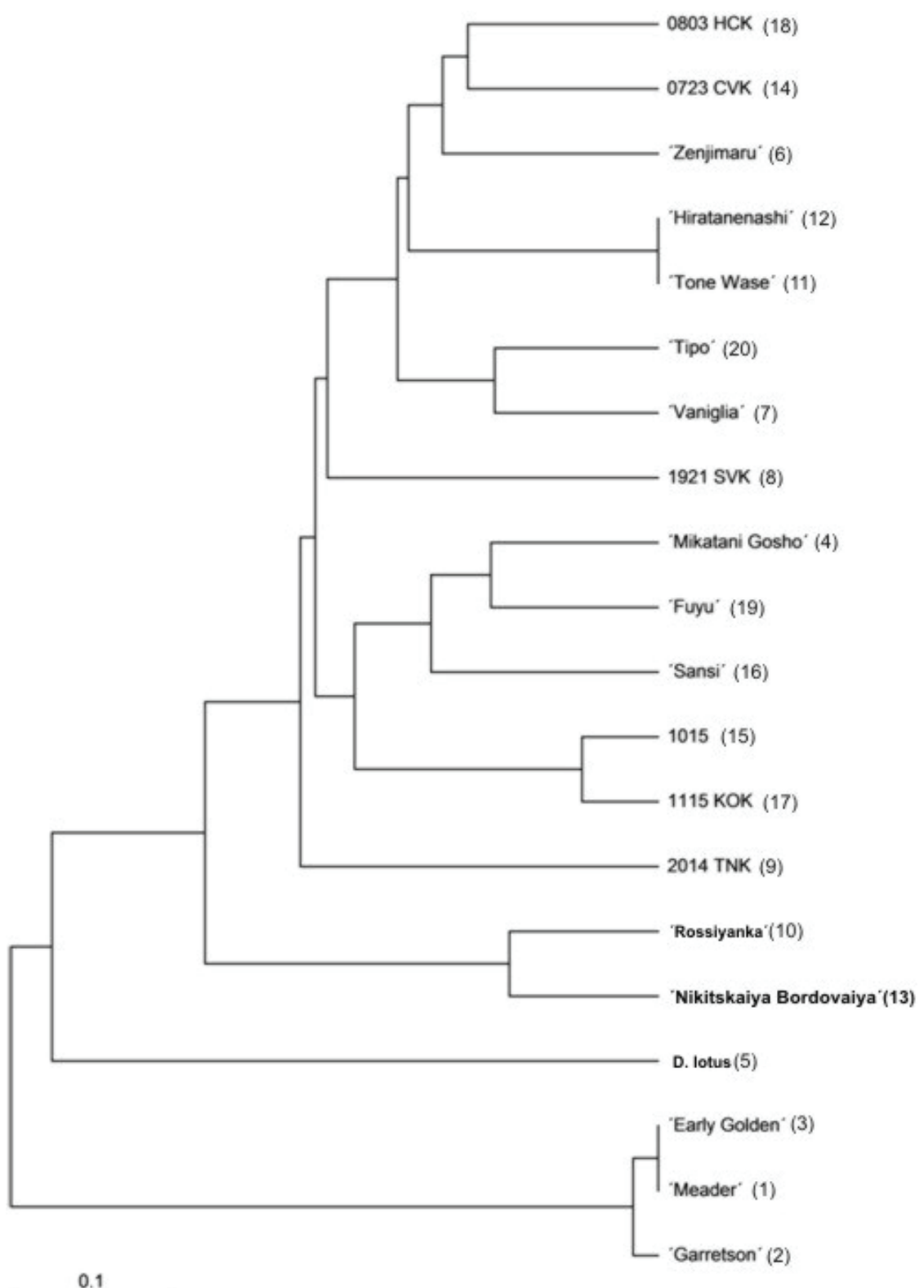

2: Dendrogram of genetic relationships of 20 persimmon cultivars (RAPD analysis); on the $x$-axis is the similarity coefficient

the picture. Analyses were carried out in order to accurately determine the individual cultivars and their assignment to the standard. The primer in Fig. 3 significantly distinguished cultivars like: 'Meader' (1), 'Garretson' (2), 'Early Golden' (3), botanical species Diospyros lotus (5), 'Rossiyanka' (10) and 'Nikitskaiya Bordovaiya' (13) from other cultivars.

The dendrogram of genetic relationships among persimmon cultivars based on $12 \mathrm{i}$-PBS primers is presented in Fig. 4.

The i-PBS dendrogram classified all the 20 cultivars into four groups. The first group includes single $D$. lotus (5), which is also clearly separated from other cultivars of the genus Diospyros. The second cluster is composed of American persimmons like 'Meader'
(1), 'Garretson' (2), 'Early Golden '(3). The third cluster includes interspecific hybrids 'Rossiyanka' (10) and 'Nikitskaiya Bordovaiya' (13), which arised from crosses of D. virginiana and D. kaki. The rest of 14 cultivars which were clustered in the last cluster belongs to group of Japanese, Italian and Chinesese persimmons. Cultivars of unknown origin 1115 KOK (17), 1015 (15), 1921 SVK (8), 2014 TNK (9), 0723 CVK (14) and 0803 HCK (18) are in the same cluster like known cultivars 'Zenjimaru' (6), 'Vaniglia' (7), 'Sansi' (16), 'Tipo' (20), 'Hiratanenashi'(12), 'Tone Wase' (11), 'Mikatani Gosho' (4), and 'Fuyu' (19) and therefore these cultivars share similar genetic background. 


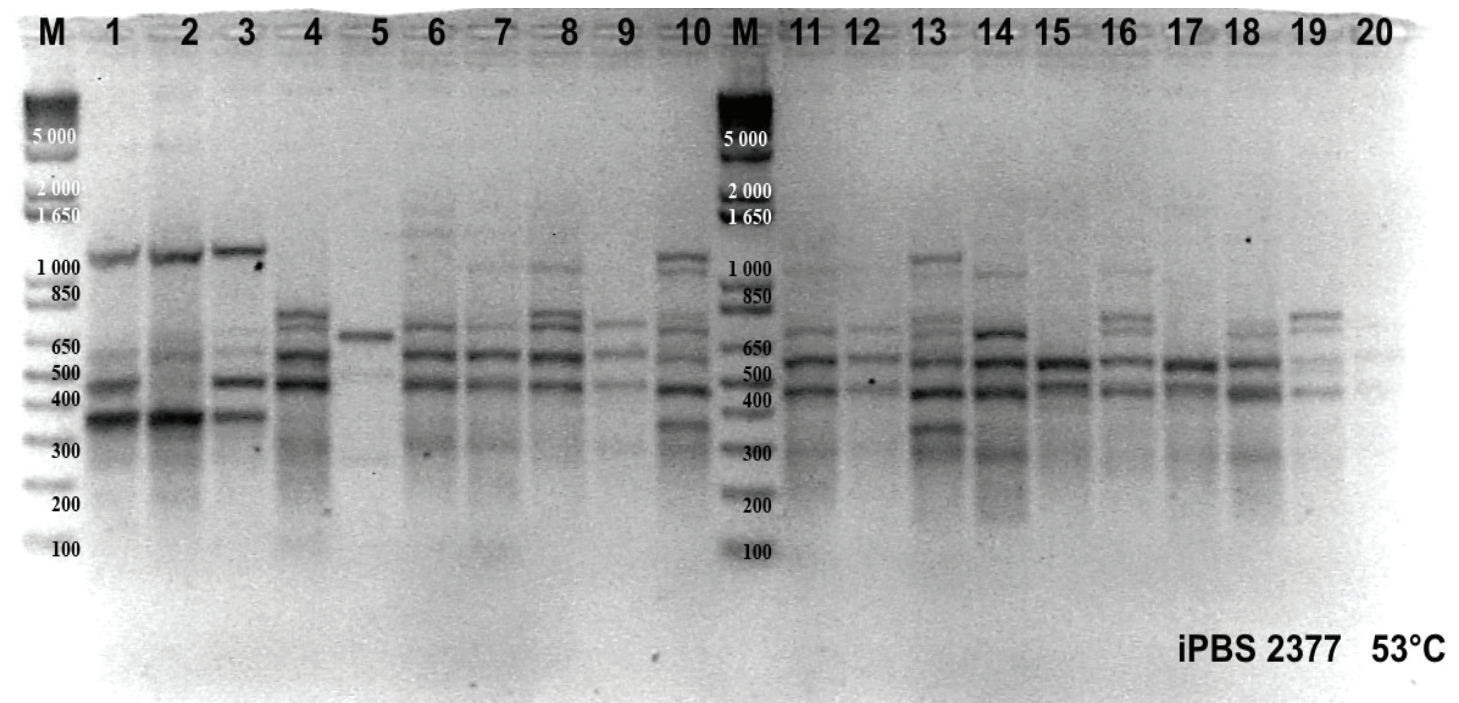

3: I-PBS analysis of all cultivars with -PBS primer 2076; 1-20 - numbers of cultivars, "M" - 100 bp DNA ladder (Life Technologies)

\section{Results of combination of both methods (RAPD and i-PBS)}

Genetic polymorphism was evaluated in 14 known cultivars and 6 cultivars of unknown origin of the genus Diospyros by using 12 i-PBS primers, 10 OPT primers and 3 OPA primers. A total of 3520 polymorphic bands were evaluated and polymorphism was assessed in 176 markers. The similarity dendrogram is presented in Fig. 5 and Fig. 6.

Distribution of cultivars in the dendrogram was very similar to previous dendrograms. The first part of dendrogram includes single botanical species Diospyros lotus (5), which is also clearly separated from other cultivars of the genus Diospyros. The second is cluster of American persimmons, where 3 cultivars of American persimmon are included: 'Meader' (1), 'Garretson' (2) and 'Early Golden' (3). This cluster was clearly separated from the rest of cultivars. The third cluster includes two interspecific hybrids 'Rossiyanka' (10) and 'Nikitskaiya Bordovaiya' (13), which originated from crosses of D. virginiana and $D$. kaki. The fourth cluster includes cultivars without significant distinction. There are fourteen cultivars belonging to Japanese, Italian and Chinesese persimmon in this cluster. Cultivars of unknown origin 1115 KOK (17), 1015 (15), 1921 SVK (8), 2014 TNK (9), 0723 CVK (14) and 0803 HCK (18) are in the same cluster like known cultivars 'Zenjimaru' (6), 'Vaniglia' (7), 'Sansi' (16), 'Tipo' (20), 'Hiratanenashi '(12),' Tone Wase' (11), 'Mikatani Gosho' (4) and 'Fuyu' (19) and therefore these cultivars share similar genetic background.

The fourth cluster is divided into several subclusters. The cultivar of unknown origin TNK 2014 (9) is separated from the other cultivars. This cultivar is the closest to pair of other cultivars of unknown origin 1115 KOK (17) and 1015 (15) and it means they are closely related. These three cultivars are genetically closest to cultivars like 'Mikatani Gosho' (4), 'Fuyu' (19) and 'Sansi' (16), which create another subcluster. Pairs of cultivars like 'Hiratanenashi' (12) and 'Tone Wase', 'Vaniglia '(7) and 'Tipo '(20) and triplet of cultivars of unknown origin like 0723 CVK (14), 0803 HCK (18) and 'Zenjimaru' (6) create last subcluster. The cultivar 1921 SVK (8) is separated from the others, which belong to this subcluster.

\section{DISCUSSION}

In the previous literature sources it is shown that the RAPD method is suitable for the study of persimmon cultivars (Nakamura and Kobayashi, 1994; Badenes et al., 2003). Badenes et al. (2003) and Yýldýz et al. (2007) used a set of primers OPA to distinguish different cultivars of persimmon. Badanes et al. (2003) used the OPA-8, OPA-18 and OPA-19. Yýldýz et al. (2007) used primer OPA-18 and OPA-19. Primers OPA-8, OPA-18 and OPA-19 were used also in this work. The OPA primers amplified from 7 to 15 polymorphic products in size from 150 to $1400 \mathrm{bp}$. In this work, an average yield of OPA primers reached 10.3 markers/ primer, which is three times more than average of 2.4 markers/primer in Badenes et al. (2003), but less than 12.6 markers/primer in Yýldýz et al. (2007). Primers OPT reached an average yield of 5.7 markers/primer and primers i-PBS reached an average yield of 7.3 markers/primer. It follows, that the primers OPA gave the most polymorphic bands.

The i-PBS technique was tested in work of Kalendar et al. (2010) in several plant and animal species. The effectiveness of this method was confirmed also by Gailite et al. (2011). According to recent literature sources, no one has tested the i-PBS method for distinguishing cultivars of persimmon. On the basis of these results it can be said that the 


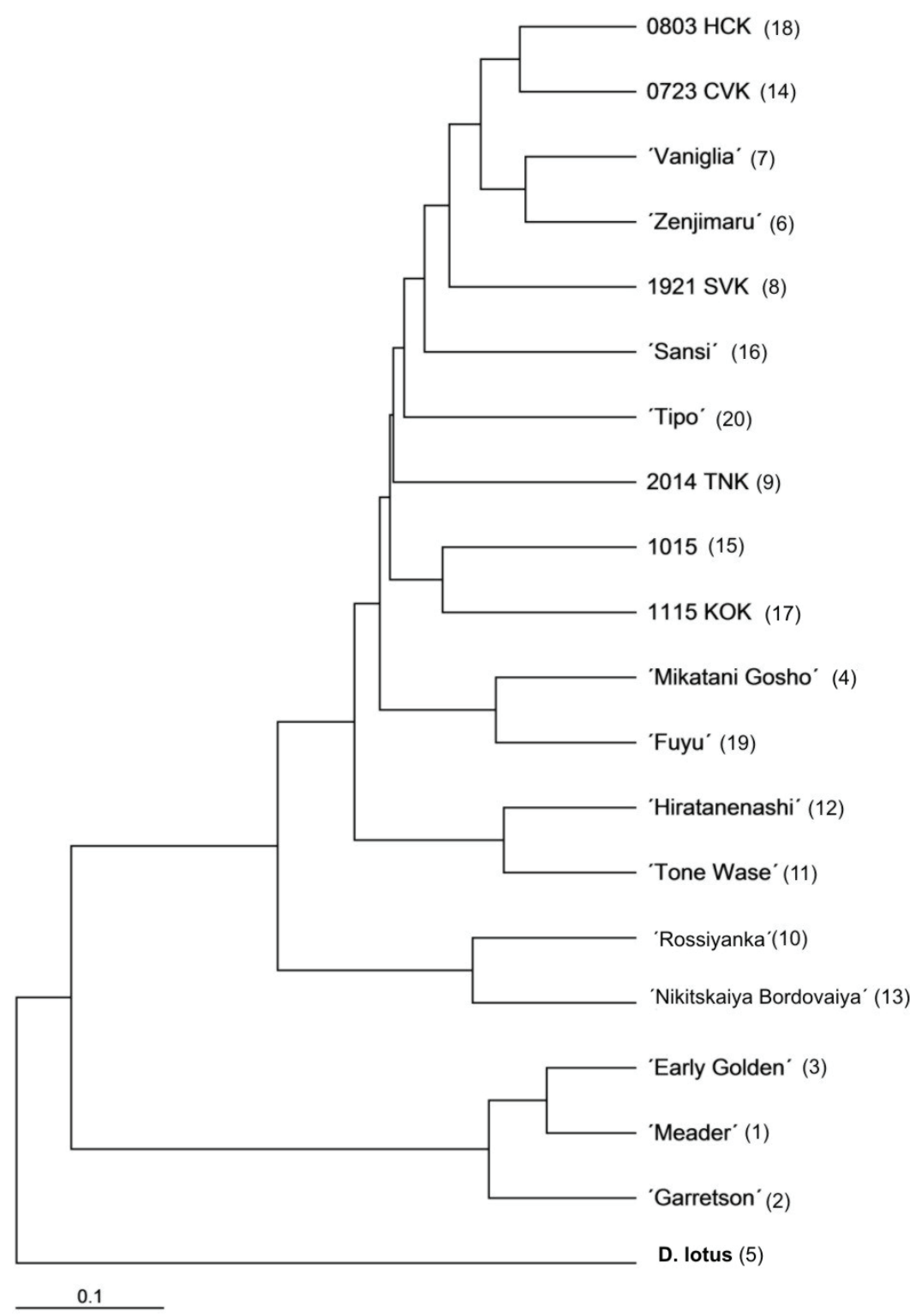

4: Dendrogram of genetic relationships of 20 persimmon cultivars ( $i$-PBS analysis); on the $x$-axis is the similarity coefficient

persimmon cultivars have a relatively high level of genetic diversity.

Earlier studies using RAPD in Badenes et al. (2003); Akbulut et al. (2008) showed large genetic variation between different cultivars of D. kaki. In the papers of Thaipong et al. (2003) and Guo and Luo (2011) cultivars of Japanese and American persimmon were significantly separated. It was also confirmed in this work. Cultivars were assigned to the cluster according to the place of origin.

The largest group is formed by cultivars of Japanese persimmon (D. kaki). Japanese cultivars ('Mikatani Gosho', 'Zenjimaru', 'Tone Wase', 'Hiratanenashi', 'Fuyu'), Chinese cultivar 'Sansi' and two Italian cultivars 'Vaniglia' and 'Tipo'. They are clustered without significant distinction. One explanation may be that the Italian cultivars have their origin in the Japanese cultivars. Yonemori et al. (2008) using the UPGMA analysis ranked cultivar 'Vaniglia' into the first group of European cultivars, compared with the Neighbor Joining analysis clustered cultivar 'Vaniglia' in the second group of European cultivars. Yonemori et al. (2008) came to the conclusion that groups of Japanese and European cultivars share a similar genetic background.

In the work of Giordani (2002) it is written that cultivar 'Tonewase' is a bud mutation of the cultivar 'Hiratanenashi', which is confirmed by the placement of these two cultivars in the dendrogram in this work.

The dendrograms created by two different methods (RAPD and i-PBS) are very similar. Both methods are "random" 


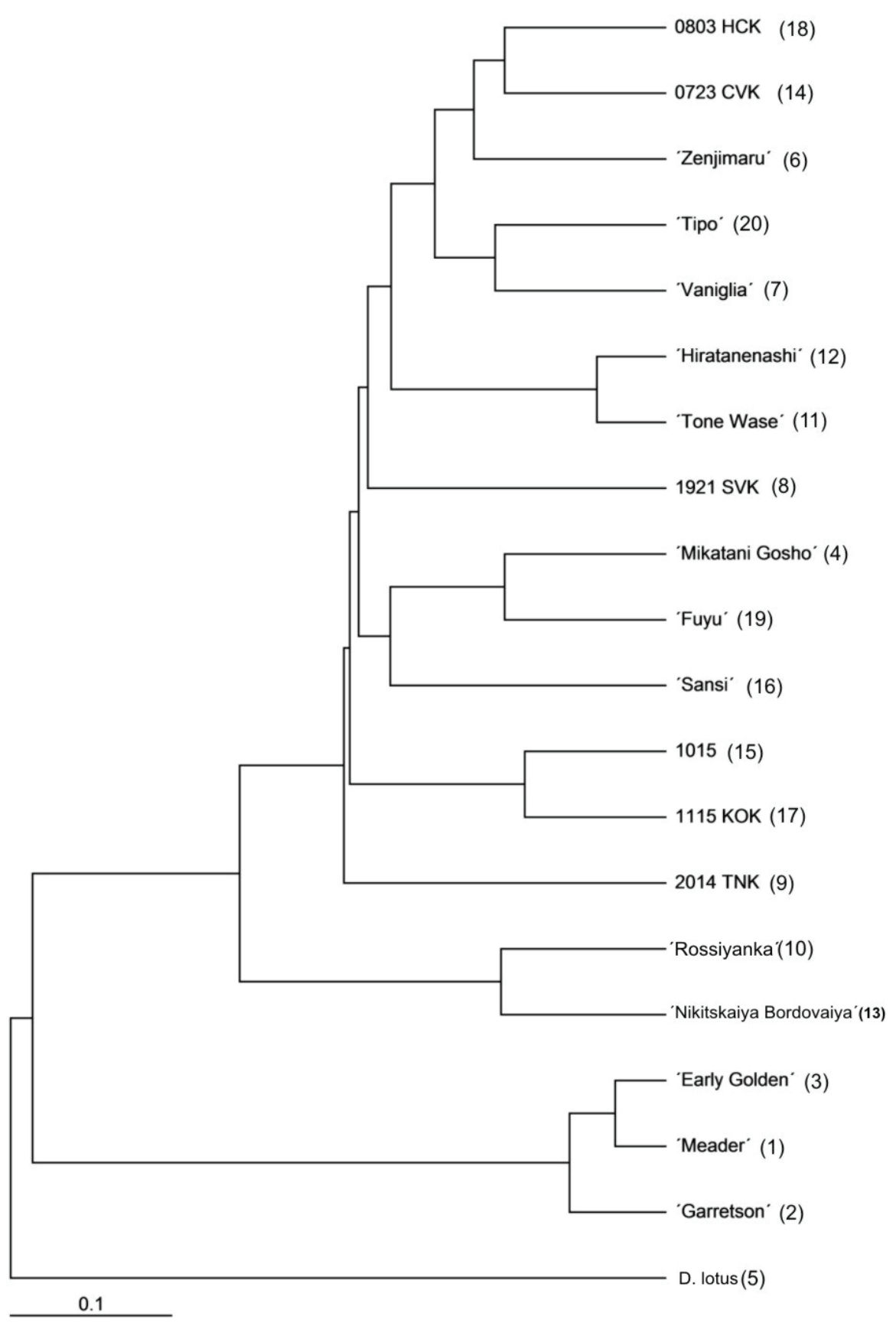

5: Dendrogram of genetic relationships of 20 persimmon cultivars (combinaton of both analyses RAPD and $i$-PBS); on the $x$-axis is the similarity coefficient

when random primers bind the target DNA. Better distinction of persimmon cultivars could be identified by using other amplification methods, such as the SSR method. Therefore, the analysis could follow by SSR analysis, which is more specific and reliable than RAPD. According to Guo and Luo (2011) the SSR analysis presents new opportunities for studying genetic diversity of Japanese persimmon.

Molecular methods present unlimited access to the source of genetic variation. In addition, we can discover some genotypic differences of which distinction by other methods is difficult or impossible.

\section{CONCLUSION}

Fourteen known cultivars and six cultivars of unknown origin of the genus Diospyros were subjected to RAPD and i-PBS analyses.

Basic screening of OPT primers was performed and subsequently 10 selected primers OPT and 3 primers OPA previously described in the literature were used to evaluate polymorphism in 88 markers. Altogether 1760 polymorphic bands were evaluated 


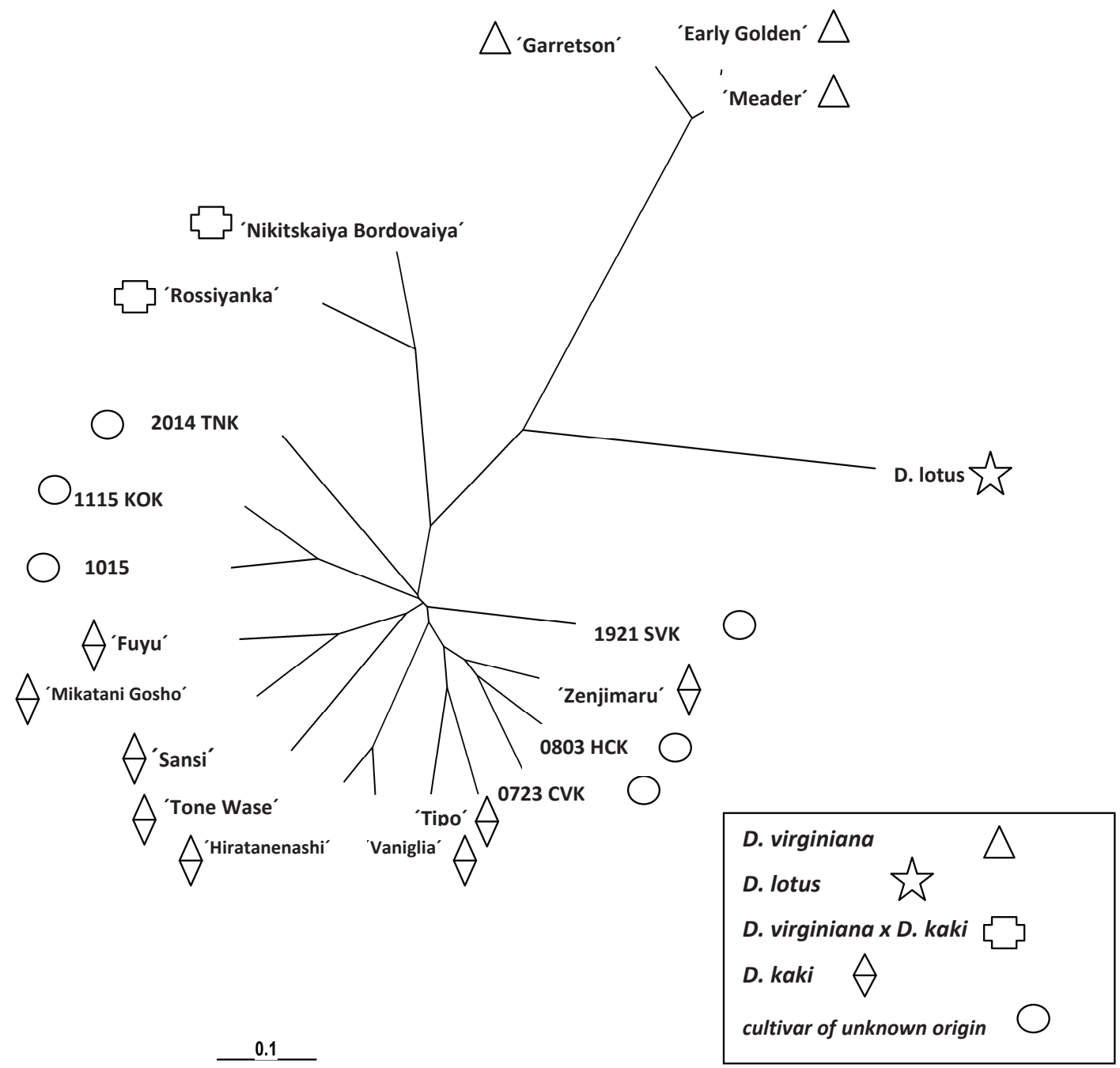

6: Unrooted dendrogram of 20 persimmon cultivars (combinaton of both analyses RAPD and $i$-PBS); on the $x$-axis is the similarity coefficient

and the size of the amplified fragments ranged from $150 \mathrm{bp}$ to $2100 \mathrm{bp}$.

Twelve i-PBS primers determined 1760 polymorphic bands scored in 88 markers and primers generated from 4 to 12 polymorphic bands. The size of the amplified fragments ranged from 100 bp to $2000 \mathrm{bp}$. A total of 3520 polymorphic bands were generated and polymorphism was assessed in 176 markers.
All the dendrograms clearly separated the assessed cultivars into 4 clusters. It is obvious, that the unknown cultivars belonging to the cluster of Japanese persimmon (D. kaki). According to this study the RAPD and i-PBS were reliable enough to detect differences between the genetically close cultivars of persimmon.

\section{SUMMARY}

Identification of persimmon cultivars is one of the main problems in the present due to misleading transliterations, local names, synonyms and homonyms. The RAPD andi-PBS techniques were used to study genetic diversity within the persimmon collection at Horticulture Faculty of Mendel University in Lednice. The aim of the work was to distinguish between 14 known and 6 persimmon cultivars of unknown origin. The basic screening of 20 OPT primers was applied to 4 cultivars differring in the place of origin. Those of screened primers within the group there were chosen, which gave polymorphic repeatable strong and middle strong bands. The total 10 OPT primers (OPT-01, OPT-03, OPT-09, OPT-11, OPT-12, OPT-16, OPT-17, OPT-18, OPT-19, OPT-20) were selected for further work after screening, visual and statistical evaluation for the RAPD analysis within the whole persimmon 
collection. Three OPA (OPA-8, OPA-18, OPA-19) primers previously described in the literature were also used for the RAPD reactions within the whole persimmon collection. Further 16 i-PBS, primers previously described in the literature, were also used for i-PBS analysis of the whole group of cultivars. The amplification was successful with 12 i-PBS primers. The FreeTree software package was used to generate similarity matrix and then to produce a dendrogram using UPGMA analysis.

The group of RAPD primers selected during the basic screening distinguished all the analysed cultivars. The OPT primers created a total of 1140 polymorphic bands scored in 57 markers. Selected primers generated from 3 to 11 polymorphic products in the size range of $220 \mathrm{bp}$ to $2100 \mathrm{bp}$. The OPA primers made a total of 620 polymorphic bands scored in 31 markers. Each primer generated from 7 to 15 polymorphic bands. The size of the amplified fragments ranged from $150 \mathrm{bp}$ to $1400 \mathrm{bp}$. In total, 1760 polymorphic bands in 88 markers were generated by RAPD primers (OPT and OPA) and the size of the amplified fragments ranged from $150 \mathrm{bp}$ to $2100 \mathrm{bp}$. The OPA primers reached an average yield of 10.3 markers/primer and the OPT primers had an average yield of 5.7 markers/ primer. The dendrogram of genetic relationships among persimmon cultivars based on RAPD primers is presented in Fig. 2. The RAPD dendrogram classified all the 20 cultivars into four groups. First is a cluster of American persimmons, where 3 cultivars of American persimmon are included: 'Meader' (1), 'Garretson' (2) and 'Early Golden' (3). They are representatives of D. virginiana. This cluster was clearly separated from the rest of cultivars, while cultivars 'Meader' (1) and 'Early Golden' (3) were not distinguished using the RAPD markers. The second part of dendrogram includes single botanical species Diospyros lotus (5), which is also clearly separated from other cultivars of the genus Diospyros. The third cluster includes interspecific hybrids 'Rossiyanka' (10) and 'Nikitskaiya Bordovaiya' (13), which arised from crosses of D. virginiana and D. kaki. The remaining and largest part creates a cluster of cultivars without significant distinction. This cluster includes fourteen cultivars belonging to the species D. kaki. Cultivars of unknown origin 1115 KOK (17), 1015 (15), 1921 SVK (8), 2014, TNK (9), 0723 CVK (14) and 0803 HCK (18) are in the same cluster like known cultivars 'Zenjimaru' (6), 'Vaniglia' (7), 'Sansi' (16), 'Tipo' (20), 'Hiratanenashi' (12),'Tone Wase' (11), 'Mikatani Gosho' (4) and 'Fuyu' (19) and therefore these cultivars share similar genetic background.

Twelve i-PBS primers created a total of 1760 polymorphic bands scored in 88 markers and generated distinctive 4 to 12 polymorphic bands products in the size range of $100 \mathrm{bp}$ to $2000 \mathrm{bp}$. Primers i-PBS reached an average yield of 7.3 markers/primer. The i-PBS dendrogram classified all the 20 cultivars into four groups. The first group includes single D. lotus (5), which is also clearly separated from other cultivars of the genus Diospyros. The second cluster is composed of American persimmons like 'Meader' (1), 'Garretson' (2), 'Early Golden '(3). The third cluster includes interspecific hybrids 'Rossiyanka' (10) and 'Nikitskaiya Bordovaiya' (13), which arised from crosses of D. virginiana and D. kaki. The rest of 14 cultivars which were clustered in the last cluster belongs to group of Japanese, Italian and Chinesese persimmons. Cultivars of unknown origin 1115 KOK (17), 1015 (15), 1921 SVK (8), 2014 TNK (9), 0723 CVK (14) and 0803 HCK (18) are in the same cluster like known cultivars 'Zenjimaru' (6), 'Vaniglia' (7), 'Sansi' (16), 'Tipo' (20), 'Hiratanenashi'(12), 'Tone Wase' (11), 'Mikatani Gosho' (4), and 'Fuyu' (19) and therefore these cultivars share similar genetic background.

Further genetic polymorphism was evaluated in 14 known cultivars and 6 cultivars of unknown origin of the genus Diospyros by using 12 i-PBS primers, 10 OPT primers and 3 OPA primers. A total of 3520 polymorphic bands were evaluated and polymorphism was assessed in 176 markers. Distribution of cultivars in the dendrogram was very similar to previous dendrograms. First part of dendrogram includes single botanical species Diospyros lotus (5), which is also clearly separated from other cultivars of the genus Diospyros. The second is cluster of American persimmons, where 3 cultivars of American persimmon are included: 'Meader' (1), 'Garretson' (2) and 'Early Golden' (3). This cluster was clearly separated from the rest of cultivars. The third cluster includes two interspecific hybrids 'Rossiyanka' (10) and 'Nikitskaiya Bordovaiya' (13), which originated from crosses of D. virginiana and D. kaki. The fourth cluster includes cultivars without significant distinction. There are fourteen cultivars belonging to Japanese, Italian and Chinesese persimmon in this cluster. Cultivars of unknown origin 1115 KOK (17), 1015 (15), 1921 SVK (8), 2014 TNK (9), 0723 CVK (14) and 0803 HCK (18) are in the same cluster like known cultivars 'Zenjimaru' (6), 'Vaniglia' (7), 'Sansi' (16), 'Tipo' (20), 'Hiratanenashi '(12),' Tone Wase' (11), 'Mikatani Gosho' (4) and 'Fuyu' (19) and therefore these cultivars share similar genetic background.

According to this study the RAPD and i-PBS were reliable enough to detect differences between the genetically close cultivars of persimmon. The similarities and the differences revealed among incorporation of cultivars into groups were compared with the literature findings. 


\section{REFERENCES}

AKBULUT, M., ERCISLI, S., YILDIRIM, N., ORHAN, E., AGAR, G., 2008: The comparison of persimmon genotypes (Diospyros kaki Thunb.) by using RAPD and FAME data. Romanian Biotechnological Letters, 13(4): 3851-3858.

BADENES, M., GARCÉS, A., ROMERO, C., ROMERO, M., CLAVÉ, J., ROVIRA, M., LLÁCER, G., 2003: Genetic diversity of introduced and local Spanish persimmon cultivars revealed by RAPD markers. Genetic Resources and Crop Evolution 50: 579-585.

BADENES, M. L., MARTÍNEZ-CALVO, J., LLÁCER, G., 1998: Analysis of peach germplasm from Spain. Acta Hort., 465: 243-250.

BARTOLOZZI, F., WARBURTON, M. L., ARULSEKAR, S., GRADZIEL, T. M., 1998: Genetic characterization and relatedness among California almond cultivars and breeding lines detected by randomly amplified polymorphic DNA (RAPD) analysis. J. Amer. Soc. Hort. Sci. 123(3): 381-387.

BELLINI, E., BENELLI, C., GIORDANI, E., PAFFETTI, D., PERRIA, R., 2003: Genetic and morphological relationships between possible Italian and ancestral cultivars of persimmon. Acta Hort. 601: 192-197.

BELLINI, E., GIORDANI, E., 2003: Germplasm conservation of persimmon in Europe, Acta Hort. 601: 37-46.

BOONPRAKOB, U., BYRNE, D. H., GRAHAM, C. J., OKIE, W. R., BECKMAN, T., SMITH, B. R., 2001: Genetic relationships among cultivated diploid plums and their progenitors as determined by RAPD markers. J. Amer. Soc. Hort. Sci. 126(4): 451-461.

ERCISLI, S., AGAR, G., YILDIRIM, N., ESITKEN, A., ORHAN, E., 2009: Identification of apricot cultivars in Turkey (Prunus armeniaca L.) using RAPD markers. Romanian Biotechnological Letters, 14(4): 4582-4588.

FAOSTAT - Food and agriculture organization of the United Nations; [online]. [cit. 2012-01-18] available on: http://faostat.fao.org/.

GAILITE, A., IEVINSH, G., RUNGIS, D., 2011: Genetic diversity analysis of Latvian and Estonian Saussurea esthonica populations. Environmental and Experimental Biology, 9: 115-119.

GIORDANI, E., 2002: Varietal assortment of persimmon in the countries of the Mediterranean area and genetic improvement, [online]. [cit. 2012-01-17]: http://ressources.ciheam.org/om/ pdf/a51/02600060.pdf.

GUO, D. L.; LUO, Z. R., 2011: Genetic relationships of the Japanese persimmon Diospyros kaki (Ebenaceae) and related species revealed by SSR analysis. Genetics and Molecular Research 10(2): 1060-1068.

HAMPL, V., PAVLÍČEK, A., FLEGR, J., 2001: Construction and bootstrap analysis of DNA fingerprinting-based phylogenetic trees with a freeware program FreeTree: application to trichomonad parasites. International Journal of Systematic and Evolutionary Microbiology, 51(3): 731-735.

JI Q., ZENG J., GUO Y., 201l: Using optimized random amplified polymorphic DNA (RAPD) markers to identify the category status of Citrus nobilis Lour. Gonggan, African Journal of Biotechnology 10(64): 13982-13990.

KALENDAR, R., ANTONIUS, K., SMÝKAL, P., SCHULMAN,A.H., 2010:iPBS: a universal method for DNA fingerprinting and retrotransposon isolation. Theor. Appl. Genet. 121(8): 1419-1430.

KALENDAR, R., 2011: The use of retrotransposonbased molecular markers to analyze genetic diversity, Field Veg. Crop Res. 48(2): 261-274.

KALENDAR, R., FLAVELL, A. J., ELLIS, T. H. N., SJAKSTE, T., MOISY, C., SCHULMAN, A.H., 201l: Analysis of plant diversity with retrotransposonbased molecular markers. Heredity 106(4): 520530.

KOLLER, B., LEHMANN, A., MCDERMOTT, J. M., GESSLER, C., 1993: Identification of apple cultivars using RAPD markers. Theor. Appl. Genet. 85(6-7): 901-904.

KUMAR, A., BENNETZEN, J. L., 1999: Plant retrotransposons. Annual Review Genetics, 34: 479-532.

MONDINI, L., NOORANI, A., PAGNOTTA, M. A., 2009: Assessing plant genetic diversity by molecular tools, Diversity 1(1): 19-35, ISSN 14242818.

NAKAMURA, Y., KOBAYASHI, S., 1994: DNA Restriction Fragment Length Variability in Diospyros kaki and Related Diospyros Species. HortScience, 29(7): 809-811.

NEI, M., 1972: Genetic distance between populations. Am Nat. 106: 283-292.

ONDRÁŠEK, I., KRŠKA, B., 2009: Growing and possibilities of introduction of some species of genus Diospyros in the Czech Republic. Acta Horticulturae, 833: 47-50, ISSN 0567-7572.

PAGE, R. D. M., 1996: TREEVIEW: An application to display phylogenetic trees on personal computers. Computer Applications in the Biosciences 12: 357-358.

PRAKASH, D.P., NARAYANASWAMY, P., SONDUR, S. N., 2002: Analysis of molecular diversity in guava using RAPD markers. J. Hort. Sci. Biotech., 77(3): 287-293.

RADDOVÁ, J., Aplikace metod založených na amplifikaci DNA při studiu genomu rodu Prunus, 2005, Disertační práce, MZLU Brno, ZF Lednice.

SUGIURA, A., 2005: Retrospects and prospects on persimmon research. Acta Horticulturae 685: 177186. ISSN 0567-7572.

THAIPONG, K., KRISANAPOOK, K., BOONPRAKOB, U., 2003: Evaluation of genetic relationships of persimmons in Thailand based on RAPD markers. Thai J. Agric. Sci. 36(1): 9-20.

ULANOVSKY, S., GOGORCENA, Y., DE TODA, F. M. ORTIZ, J. M., 2002: Use of molecular markers in detection of synonymies and homonymies in 
grapevines (Vitis vinifera L.). Scientia Horticulturae 92(2/4): 241-254.

WILLIAMS, J. G. K., KUBELIK, A. R., LIVAK, K. J., RAFALSKI, J. A., TINGEY, S. V., 1990: DNA polymorphisms amplified by arbitrary primers are useful as genetic markers. Nucleic Acids Res. 18(22): 6531-6535.

YAKUSHIJI, H., NAKATSUKA, A., 2007: Recent persimmon research in Japan. Japanese Journal of Plant Science, 42-62 [online]. [cit. 2012-02-13] available on: http://www.globalsciencebooks.info/ JournalsSup/images/0712/JJPS_1\%282\%294262o.pdf.

YAMAGISHI, M., MATSUMOTO, S., NAKATSUKA, A., ITAMURA, H., 2005: Identification of persimmon (Diospyros kaki) cultivars and phenetic relationships between Diospyros species by more effective RAPD analysis. Scientia Horticulturae, 105: 283-290.

YEH, F. C., BOYLE, T. J. B., 1997: Population genetic analysis of codominant and dominant markers and quantitative traits. Belgian Journal of Botany 129: 157.

YONEMORI, K., HONSHO, CH., KITAJIMA, A., ARADHYA. M., GIORDANI, E., BELLINI, E., PARFITT D. E., 2008: Relationship of European persimmon (Diospyros kaki Thunb.) cultivars to Asian cultivars, characterized using AFLPs, Genet Resour Crop Evol 55(1): 81-89.

YONEMORI, K., SUGIURA, A., YAMADA, M., 2000: Persimmon genetics and breeding. Plant Breeding Reviews, 19: 191-225, ISBN 0-471-38787-8.

YÝLDÝZ, M., BAYAZIT, S., CEBESOY, S., ARAS., S., 2007: Molecular diversity in persimmon (Diospyros kaki L.) cultivars growing around Hatay province in Turkey. African Journal of Biotechnology Vol. 6 (20): 2393-2399.

ZAHNG, Q., GUO, D., LUO, Z., 2009: Identification and Taxonomic Status of Chinese Diospyros spp. (Ebenaceae) Androecious Germplasms. Acta Horticulture, 833: 91-96.

Mgr. Jana Raddová, Ph.D., Ing. Hana Ptáčková, Ing. Jana Čechová, Ústav genetiky - Mendeleum, Ing. Ivo Ondrášek, Ph.D., Ústav ovocnictví, Mendelova univerzita v Brně, Valtická 334, Lednice 69144, Česká republika, e-mail: radj@zf.mendelu.cz 